\title{
Semi Implicit Hybrid Methods with Higher Order Dispersion for Solving Oscillatory Problems
}

\author{
S. Z. Ahmad, ${ }^{1}$ F. Ismail, ${ }^{2}$ N. Senu, ${ }^{2}$ and M. Suleiman ${ }^{2}$ \\ ${ }^{1}$ Department of Mathematics, Universiti Putra Malaysia, 43400 Serdang, Selangor, Malaysia \\ ${ }^{2}$ Department of Mathematics and Institute for Mathematical Research, Universiti Putra Malaysia, 43400 Serdang, Selangor, Malaysia
}

Correspondence should be addressed to S. Z. Ahmad; sufia_zulfa@yahoo.com

Received 11 January 2013; Accepted 4 March 2013

Academic Editor: Juan Carlos Cortés López

Copyright ( $) 2013$ S. Z. Ahmad et al. This is an open access article distributed under the Creative Commons Attribution License, which permits unrestricted use, distribution, and reproduction in any medium, provided the original work is properly cited.

\begin{abstract}
We constructed three two-step semi-implicit hybrid methods (SIHMs) for solving oscillatory second order ordinary differential equations (ODEs). The first two methods are three-stage fourth-order and three-stage fifth-order with dispersion order six and zero dissipation. The third is a four-stage fifth-order method with dispersion order eight and dissipation order five. Numerical results show that SIHMs are more accurate as compared to the existing hybrid methods, Runge-Kutta Nyström (RKN) and RungeKutta (RK) methods of the same order and Diagonally Implicit Runge-Kutta Nyström (DIRKN) method of the same stage. The intervals of absolute stability or periodicity of SIHM for ODE are also presented.
\end{abstract}

\section{Introduction}

Second-order ordinary differential equations (ODEs) which are oscillatory in nature often arise in many scientific areas of engineering and applied sciences such as celestial mechanics, molecular dynamics, and quantum mechanics. Consider the numerical solution of the initial value problem (IVP) for second-order ODEs in the form

$$
y^{\prime \prime}=f(t, y), \quad y\left(t_{0}\right)=y_{0}, \quad y^{\prime}\left(t_{0}\right)=y_{0}^{\prime},
$$

in which the first derivative does not appear explicitly. Apparently, some of the most common methods used for solving second-order ODEs numerically are Runge-Kutta Nyström (RKN) and Runge-Kutta methods for Runge-Kutta method the IVPs need to be reduced to a system of first-order ODEs twice the dimension. The IVP can be solved using a particular explicit hybrid algorithms which were developed by Franco [1] or a multistep method for special second-order ODEs as in Yap et al. [2]. Franco [3] proposed that (1) can be solved using a particular explicit hybrid algorithms or special multistep methods for second-order ODEs. Franco [3] constructed explicit two-step hybrid methods of order four up to order six for solving second-order IVPs by considering the local truncation error and order conditions developed by Coleman [4].

Most of the IVPs represented by (1) have solutions which are oscillatory in nature, making it difficult to get the accurate numerical results. To address the problem several authors [5-7] focused their research on developing methods with reduced phase lag and dissipation, where phase-lag or dispersion error is the difference of the angle for the computed solution and the exact solution and dissipation is the distance of the computed solution from the standard cyclic solution. The analysis of phase-lag or dispersion errors was first introduced by Brusa and Nigro [8]. Van der Houwen and Sommeijer [9] proposed explicit RKN methods of order four, five, and six with reduced phase-lag of order $q=6,8,10$, respectively. Senu et al. $[7,10]$ developed diagonally implicit RKN method with dispersion of higher order for solving oscillatory problems. In a related work Kosti et al. [11] constructed an optimized RKN method (OPRKN) based on the existing explicit four-stage fifth-order RKN method for the integration of oscillatory IVPs. In his derivation he used the phase-lag, amplification factor and the first derivative of the amplification factor by equating them to zero. Later, Kosti et al. [12] also developed an OPRKN method based on the same explicit RKN method, in which he used the phase lag, amplification factor, and the first 
derivative of the phase-lag properties instead of using only the first derivative of amplification factor in his previous work.

In this paper, we constructed three-stage fourth-order and three-stage fifth-order methods with dispersion order six and zero dissipation and also four-stage fifth-order method with dispersion order eight and dissipation order five. It is done by taking the dispersion relations for the semiimplicit hybrid methods and solving them together with the algebraic conditions of the methods. The intervals of stability of the methods are also presented. Finally, numerical tests on second-order differential equation for oscillatory problems are given.

\section{Analysis Phase Lag of the Methods}

An $s$-stage two-step hybrid method for the numerical integration of the $\operatorname{IVP}(1)$ is of the form

$$
\begin{gathered}
Y_{i}=\left(1+c_{i}\right) y_{n}-c_{i} y_{n-1} \\
+h^{2} \sum_{j=1}^{s} a_{i j} f\left(t_{n}+c_{j} h, Y_{j}\right), \\
i=1, \ldots, s, \\
y_{n+1}=2 y_{n}-y_{n-1}+h^{2} \sum_{i=1}^{s} b_{i} f\left(t_{n}+c_{i} h, Y_{i}\right),
\end{gathered}
$$

where $b_{i}, c_{i}$, and $a_{i j}$ can be represented in Butcher notation by the table of coefficients as follows:

$$
\begin{array}{c|c|ccc}
c & A \\
\hline & b^{T}
\end{array}=\begin{array}{cccc}
c_{1} & a_{1,1} & \cdots & a_{1, s} \\
\vdots & \vdots & \ddots & \vdots \\
c_{s} & a_{s, 1} & \cdots & a_{s, s} \\
\hline & b_{1} & \cdots & b_{s} .
\end{array}
$$

The methods of the form (2) are defined by

$$
\begin{gathered}
Y_{1}=y_{n-1}, \quad Y_{2}=y_{n}, \\
Y_{i}=\left(1+c_{i}\right) y_{n}-c_{i} y_{n-1} \\
+h^{2} \sum_{j=1}^{i} a_{i j} f\left(t_{n}+c_{j} h, Y_{j}\right), \\
i=3, \ldots, s, \\
y_{n+1}=2 y_{n}-y_{n-1} \\
+h^{2}\left[b_{1} f_{n-1}+b_{2} f_{n}+\sum_{i=3}^{s} b_{i} f\left(t_{n}+c_{i} h, Y_{i}\right)\right],
\end{gathered}
$$

where $f_{n-1}=f\left(t_{n-1}, y_{n-1}\right), f_{n}=f\left(t_{n}, y_{n}\right), h=\Delta t=t_{n+1}-t_{n}$ and the first two nodes are $c_{1}=-1$ and $c_{2}=0$. The method only requires to evaluate $f\left(t_{n}, y_{n}\right), f\left(t_{n}+c_{3} h, Y_{3}\right), \ldots, f\left(t_{n}+\right.$ $\left.c_{s} h, Y_{s}\right)$ for each step after the starting procedure. This method is considered as a two-step hybrid method with $s-1$ stages per step and the semi-implicit hybrid with the diagonal elements being equal can be written in Butcher tableau as follows:

$$
\begin{array}{c|ccccc}
-1 & & & & & \\
0 & & & & & \\
c_{3} & a_{3,1} & a_{3,2} & \gamma & & \\
\vdots & \vdots & \vdots & \ddots & \gamma & \\
c_{s} & a_{s, 1} & a_{s, 2} & \cdots & a_{s, s-1} & \gamma \\
\hline & b_{1} & b_{2} & \cdots & b_{s-1} & b_{s} .
\end{array}
$$

Phase analysis can be divided into two parts. First is the inhomogeneous part, in which the phase error is constant in time and second is the homogeneous one, in which the phase errors are accumulated as $n$ increases. As proposed by Franco [3], the phase analysis is investigated using the second-order homogeneous linear test model

$$
y^{\prime \prime}(t)=-\lambda^{2} y(t) \quad \text { for } \lambda>0, \lambda \in \Re .
$$

Alternatively when (2) are applied to (8), they can be written in vector form as

$$
\begin{gathered}
\mathbf{Y}=(\mathbf{e}+\mathbf{c}) y_{n}-\mathbf{c} y_{n-1}-H^{2} \mathbf{A Y} \\
y_{n+1}=2 y_{n}-y_{n-1}-H^{2} \mathbf{b}^{\mathbf{T}} \mathbf{Y}
\end{gathered}
$$

where $H=\lambda h, \mathbf{Y}=\left(Y_{1}, \ldots, Y_{s}\right)^{T}, \mathbf{c}=\left(c_{1}, \ldots, c_{s}\right)^{T}$, and $\mathbf{e}=$ $(1, \ldots, 1)^{T}$. From (9) we obtain

$$
\mathbf{Y}=\left(\mathbf{I}+H^{2} \mathbf{A}\right)^{-1}(\mathbf{e}+\mathbf{c}) y_{n}-\left(\mathbf{I}+H^{2} \mathbf{A}\right)^{-1} \mathbf{c} y_{n-1} \text {. }
$$

Substituting (11) into (10), the following recursion relation is obtained:

$$
y_{n+1}-S\left(H^{2}\right) y_{n}+P\left(H^{2}\right) y_{n-1}=0,
$$

where

$$
\begin{gathered}
S\left(H^{2}\right)=2-H^{2} \mathbf{b}^{\mathbf{T}}\left(\mathbf{I}+H^{2} \mathbf{A}\right)^{-1}(\mathbf{e}+\mathbf{c}), \\
P\left(H^{2}\right)=1-H^{2} \mathbf{b}^{\mathbf{T}}\left(\mathbf{I}+H^{2} \mathbf{A}\right)^{-1} \mathbf{c} .
\end{gathered}
$$

Solving the difference system (12), the computed solution is given by

$$
y_{n}=2|c||\rho|^{n} \cos (\omega+n \phi),
$$

where $\rho$ is the amplification factor, $\phi$ is the phase, $\omega$ and $c$ are real constants determined by $y_{0}$ and $y_{0}^{\prime}$ and the hybrid parameters. The exact solution of (8) is given by

$$
y\left(t_{n}\right)=2|\sigma| \cos (\chi+n H),
$$

where $n$ is the number of term, $\sigma$ and $\chi$ are real constants determined by initial conditions. Equations (14) and (15) led to the following definition.

Definition 1 (phase lag). Apply the hybrid method (2) to (8). Next we define the phase lag $\varphi(H)=H-\phi$. If $\varphi(H)=$ $O\left(H^{q+1}\right)$, and then the hybrid method is said to have phaselag order $q$. Additionally, the quantity $d(H)=1-|\rho|$ is called amplification error. If $d(H)=O\left(H^{r+1}\right)$, then the hybrid method is said to have dissipation order $r$. 
From Definition 1, it follows that

$$
\begin{gathered}
\varphi(H)=H-\cos ^{-1}\left(\frac{S\left(H^{2}\right)}{2 \sqrt{P\left(H^{2}\right)}}\right), \\
d(H)=1-\sqrt{P\left(H^{2}\right)}
\end{gathered}
$$

Let us denote $S\left(H^{2}\right)$ and $P\left(H^{2}\right)$ to be the following:

$$
\begin{aligned}
& S\left(H^{2}\right)=\frac{2+\sum_{i=1}^{s-1} \alpha_{i} H^{2 i}}{\left(1+\gamma H^{2}\right)^{s-2}}, \\
& P\left(H^{2}\right)=\frac{1+\sum_{i=1}^{s-1} \beta_{i} H^{2 i}}{\left(1+\gamma H^{2}\right)^{s-2}} .
\end{aligned}
$$

Based on the definition of phase lag, the dispersion relations are developed. For a zero dissipative method with three-stage $(s=3)$, the dispersion relation of order six $(q=6)$ is given by the following:

Order 6:

$$
\alpha_{2}=\frac{1}{\gamma}\left(\frac{1}{360}-\gamma^{2}\right),
$$

and the dispersion relations up to order eight for $s=4$ are given by

Order 6:

$\beta_{3}-2 \beta_{2} \gamma-\alpha_{3}+\gamma \alpha_{2}-\frac{1}{2} \beta_{2}=-\frac{3}{2} \gamma^{2}+\frac{1}{360}-2 \gamma^{3}$,

Order 8:

$$
\begin{gathered}
\frac{1}{4} \beta_{2}^{2}-\left(\frac{7}{2} \gamma^{2}+\gamma+\frac{1}{24}\right) \beta_{2}-\gamma \alpha_{3}+\gamma^{2} \alpha_{2}+\left(2 \gamma+\frac{1}{2}\right) \beta_{3} \\
=\frac{1}{20160}-\frac{1}{24} \gamma^{2}-2 \gamma^{3}-\frac{13}{4} \gamma^{4} .
\end{gathered}
$$

The following quantity is used to determine that the dissipation constant of the formula for $s=3,4$ is

(i) for $s=3$

$$
\begin{aligned}
1-\sqrt{P\left(H^{2}\right)}= & \left(-\frac{1}{2} \beta_{1}+\frac{1}{2} \gamma\right) H^{2} \\
+ & \left(-\frac{1}{2} \beta_{2}+\frac{1}{4} \gamma \beta_{1}-\frac{3}{8} \gamma^{2}+\frac{1}{8} \beta_{1}^{2}\right) H^{4} \\
+ & \left(\frac{1}{4} \gamma \beta_{2}-\frac{3}{16} \gamma^{2} \beta_{1}+\frac{5}{16} \gamma^{3}+\frac{1}{4} \beta_{1} \beta_{2}\right. \\
& \left.\quad-\frac{1}{16} \gamma \beta_{1}^{2}-\frac{1}{16} \beta_{1}^{3}\right) H^{6} \\
+ & \left(\frac{5}{128} \beta_{1}^{4}-\frac{3}{16} \gamma^{2} \beta_{2}+\frac{5}{32} \gamma^{3} \beta_{1}\right. \\
& +\frac{3}{64} \gamma^{2} \beta_{1}^{2}+\frac{1}{8} \beta_{2}^{2}-\frac{3}{16} \beta_{1}^{2} \beta_{2}+\frac{1}{32} \beta_{1}^{3} \gamma \\
& \left.\quad-\frac{1}{8} \gamma \beta_{1} \beta_{2}-\frac{35}{128} \gamma^{4}\right) H^{8}+O\left(H^{10}\right),
\end{aligned}
$$

(ii) for $s=4$

$$
\begin{aligned}
1-\sqrt{P\left(H^{2}\right)}= & \left(\gamma-\frac{1}{2} \beta_{1}\right) H^{2} \\
+ & \left(\frac{1}{2} \beta_{1} \gamma-\gamma^{2}-\frac{1}{2} \beta_{2}+\frac{1}{8} \beta_{1}^{2}\right) H^{4} \\
+ & \left(-\frac{1}{2} \beta_{1} \gamma^{2}+\frac{1}{2} \beta_{2} \gamma+\gamma^{3}-\frac{1}{2} \beta_{3}-\frac{1}{8} \beta_{1}^{2} \gamma\right. \\
& \left.+\frac{1}{4} \beta_{1} \beta_{2}-\frac{1}{16} \beta_{1}^{3}\right) H^{6} \\
+ & \left(-\gamma^{4}+\frac{1}{2} \beta_{1} \gamma^{3}-\frac{1}{2} \beta_{2} \gamma^{2}\right. \\
& +\frac{1}{2} \beta_{3} \gamma-\frac{1}{4} \beta_{1} \beta_{2} \gamma+\frac{1}{8} \beta_{1}^{2} \gamma^{2} \\
& +\frac{1}{4} \beta_{1} \beta_{3}+\frac{1}{8} \beta_{2}^{2}+\frac{1}{16} \beta_{1}^{3} \gamma \\
& \left.-\frac{3}{16} \beta_{1}^{2} \beta_{2}+\frac{5}{128} \beta_{1}^{4}\right) H^{8}+O\left(H^{10}\right) .
\end{aligned}
$$

From (12), the stability polynomial of hybrid method can be written as

$$
\xi^{2}-S\left(H^{2}\right) \xi+P\left(H^{2}\right)=0 .
$$

The numerical solution defined by (12) should be periodic. The necessary conditions are

$$
P\left(H^{2}\right) \equiv 1, \quad\left|S\left(H^{2}\right)\right|<2, \quad \forall H^{2} \in\left(0, H_{p}^{2}\right),
$$

and interval $\left(0, H_{p}^{2}\right)$ is known as the periodicity interval of the method. The method is called zero dissipative when $d(H)=$ 0 , that is, if it satisfies conditions (16). Otherwise, as the method possesses a finite order of dissipation, the integration process is stable if the coefficients of polynomial in (23) satisfy the conditions

$$
\begin{array}{r}
P\left(H^{2}\right)<1, \quad\left|S\left(H^{2}\right)\right|<1+P\left(H^{2}\right), \\
\forall H^{2} \in\left(0, H_{s}^{2}\right),
\end{array}
$$

and interval $\left(0, H_{s}^{2}\right)$ is known as the interval of absolute stability of the method.

\section{Construction of the Methods}

In this section, the fourth-and fifth-order SIHMs which require only three and four stages respectively are obtained. The derivations of the methods are based on the order conditions, dispersive and dissipative error, and minimization of the error constant $C_{p+1}$ of the method. The error constant is defined by

$$
C_{p+1}=\left\|\left(e_{p+1}\left(t_{1}\right)\right), \ldots,\left(e_{p+1}\left(t_{k}\right)\right)\right\|_{2},
$$

where $k$ is the number of trees of order $p+2\left(p\left(t_{i}\right)=p+2\right)$, for the $p$ th-order method and $\left(e_{p+1}\left(t_{i}\right)\right)$ is the local truncation error defined in Coleman [4]. 
The Order conditions of hybrid method given in [4] are Order 2

$$
\sum b_{i}=1
$$

Order 3

$$
\sum b_{i} c_{i}=0
$$

Order 4

$$
\sum b_{i} c_{i}^{2}=\frac{1}{6}, \quad \sum b_{i} a_{i j}=\frac{1}{12}
$$

Order 5

$$
\sum b_{i} c_{i}^{3}=0, \quad \sum b_{i} c_{i} a_{i j}=\frac{1}{12}, \quad \sum b_{i} a_{i j} c_{j}=0,
$$

Order 6

$$
\begin{gathered}
\sum b_{i} c_{i}^{4}=0, \quad \sum b_{i} c_{i}^{2} a_{i j}=\frac{1}{30}, \quad \sum b_{i} c_{i} a_{i j} c_{j}=-\frac{1}{60} \\
\sum b_{i} a_{i j} a_{i k}=\frac{7}{120}, \quad \sum b_{i} a_{i j} c_{j}^{2}=\frac{1}{180}, \\
\sum b_{i} a_{i j} a_{j k}=\frac{1}{360} .
\end{gathered}
$$

For the method, $c_{i}$ need to satisfy

$$
\sum a_{i j}=\frac{\left(c_{i}^{2}+c_{i}\right)}{2}, \quad(i=1, \ldots, s) .
$$

3.1. SIHM with Three Stages. To derive the fourth-order SIHM method, we use the algebraic conditions up to order four (27)-(29), simplifying condition (32), zero dissipation conditions $\left(\beta_{1}=\gamma, \beta_{2}=0\right)$, and dispersion relation of order six $(q=6)$, (19). The resulting system of equations consists of five nonlinear equations with seven unknown variables to be solved. Therefore, we have two degrees of freedom. The coefficients of the methods are determined in terms of the arbitrary parameters $c_{3}$ and $a_{33}$ which are given by the expressions

$$
\begin{gathered}
b_{1}=\frac{1}{6+6 c_{3}}, \quad b_{2}=\frac{6 c_{3}-1}{6 c_{3}}, \quad b_{3}=\frac{1}{6 c_{3}\left(1+c_{3}\right)}, \\
a_{31}=-\frac{c_{3}\left(30 a_{33} c_{3}-c_{3}-1\right)}{30}, \\
a_{32}=\frac{7}{15} c_{3}^{2}+a_{33} c_{3}^{2}+\frac{7}{15} c_{3}-a_{33} .
\end{gathered}
$$

By minimizing the error constant from (26) we have $c_{3}=$ $9 / 10$ and $a_{33}=1 / 30$. This method is denoted as SIHM3(4) which is given below:

$$
\begin{array}{c|ccc}
-1 & 0 & & \\
0 & 0 & 0 & \\
\frac{9}{10} & \frac{3}{100} & \frac{19}{24} & \frac{1}{30} \\
\hline & \frac{5}{57} & \frac{22}{27} & \frac{50}{513}
\end{array}
$$

With this solution, the norms of the principal local truncation error coefficient for $y_{n}$ are given by

$$
\left\|\tau^{(5)}\right\|_{2}=1.863 \times 10^{-2}
$$

where $\left\|\tau^{(5)}\right\|_{2}$ are the error equations for the fifth-order methods. This formula has dispersive order six and zero dissipation with a dispersion constant $(13 / 302400) H^{7}+$ $\mathrm{O}\left(\mathrm{H}^{9}\right)$. The interval of periodicity of the method is $(0,2.96)$.

Meanwhile, to derive the three-stage fifth-order SIHM method, the algebraic conditions (27) to (30) and equation (32) with dispersion relation of order six $(q=6)$, equation (19), and zero dissipation conditions $\left(\beta_{1}=\gamma, \beta_{2}=0\right)$ are solved simultaneously. This involves seven equations and seven unknowns need to be solved; hence it has a unique solution. This method is denoted as SIHM3(5) whose coefficients are given below:

$$
\begin{array}{c|ccc}
-1 & 0 & & \\
0 & 0 & 0 & \\
1 & \frac{1}{30} & \frac{14}{15} & \frac{1}{30} \\
\hline & \frac{1}{12} & \frac{5}{6} & \frac{1}{12} .
\end{array}
$$

With this solution, the norm of the principal local truncation error coefficient for $y_{n}$ is given by

$$
\left\|\tau^{(6)}\right\|_{2}=1.147 \times 10^{-1},
$$

where $\left\|\tau^{(6)}\right\|_{2}$ are the error equations for the sixth-order method. This formula has dispersive order six and zero dissipation with a dispersion constant (13/302400) $H^{7}+$ $O\left(H^{9}\right)$. The interval of periodicity of the method is $(0,2.96)$.

3.2. SIHM Order Five with Four Stages. The SIHM method of order five is obtained by considering the order conditions up to order five which are (27) to (30) and (32) together with dispersion relations up to order eight, ((19) and (20)). Solving all the conditions simultaneously, and then the following family of solutions in terms of free parameters $a_{41}$ and $b_{3}$ is obtained:

$$
\begin{gathered}
a_{31}=\frac{360 a_{41} b_{3}-30 a_{41}+1}{360 b_{3}}, \\
a_{32}=\frac{-360 a_{41} b_{3}+30 a_{41}-1+360 b_{3}^{2}+330 b_{3}}{360 b_{3}}, \\
a_{42}=\frac{-360 a_{41} b_{3}+30 a_{41}-29+360 b_{3}^{2}+330 b_{3}}{30\left(12 b_{3}-1\right)}, \\
a_{33}=-b_{3}+\frac{1}{12}, \quad a_{43}=-\frac{-1+20 b_{3}}{20\left(12 b_{3}-1\right)}, \\
b_{1}=\frac{1}{12}, \quad b_{2}=\frac{5}{6}, \quad b_{4}=-b_{3}+\frac{1}{12}, \\
c_{3}=1, \quad c_{4}=1 .
\end{gathered}
$$


TABLE 1: Summary of the properties of the SIHM3(4), SIHM3(5), and SIHM4(5) methods.

\begin{tabular}{lcccccc}
\hline Method & $q$ & $r$ & $\left\|\tau^{(p+1)}\right\|_{2}$ & DPC & DSC & - \\
\hline SIHM3(4) & 6 & $\infty$ & $1.863 \times 10^{-2}$ & $13 / 302400$ & $13 / 3.92 .96)$ \\
SIHM3(5) & 6 & $\infty$ & $1.147 \times 10^{-1}$ & $13 / 302400$ & $(0,2.96)$ \\
SIHM4(5) & 8 & 5 & $9.772 \times 10^{-2}$ & $241 / 881798400$ & $277 / 44089920$ & $(0,5.75)$ \\
\hline
\end{tabular}

Note that DPC is dispersion constant, DSC is dissipation constant, P.I is periodicity interval, and S.I is stability interval.

By minimizing the error norm expression, we have $a_{41}=$ $150617 / 771120$ and $b_{3}=23 / 324$.

With this solution, the norm of the principal local truncation error coefficient for $y_{n}$ is given by

$$
\left\|\tau^{(6)}\right\|_{2}=9.772 \times 10^{-2}
$$

This fifth-order formula is dispersive order eight and dissipative order five with dispersion and dissipation constants are $(241 / 881798400) H^{9}+O\left(H^{11}\right)$ and $(277 / 44089920) H^{6}+$ $\mathrm{O}\left(H^{8}\right)$ respectively. This method is denoted as SIHM4(5), the coefficients are given below (see: The SIHM4(5) method) and the interval of absolute stability of the method is $(0,5.75)$.

$$
\begin{array}{c|ccccc}
-1 & 0 & & & \\
0 & 0 & 0 & & \\
1 & \frac{199}{38556} & \frac{1403}{1428} & \frac{1}{81} & \\
1 & \frac{150617}{771120} & \frac{18583}{28560} & \frac{17}{120} & \frac{1}{81} \\
\hline & \frac{1}{12} & \frac{5}{6} & \frac{23}{324} & \frac{1}{81} .
\end{array}
$$

Table 1 shows a comparison of the properties of the methods derived.

\section{Problems Tested and Numerical Results}

In this section, SIHM3(4) is compared with five other fourthorder methods: DIRKN three-stage fourth-order derived by Senu et al. [10], DIRKN three-stage fourth-order derived by Sommeijer [13], Classical Runge-Kutta fourth-order given in Dormand [14], explicit three-stage fourth-order hybrid method derived by Franco [3], and Classical RKN fourth order given in Hairer et al. [15]. The fifth-order methods, SIHM3(5) and SIHM4(5) are compared with four other methods: DIRKN four-stage fourth-order derived by Senu et al. [7], Classical Runge-Kutta fifth order derived by Butcher [16], explicit four-stage fifth-order hybrid method derived by Franco [3], and Classical RKN fifth-order method given from Hairer et al. [15]. All the problems were executed for $t_{\text {end }}=10^{4}$ except for Orbital problem $t_{\text {end }}=100$. The test problems used are listed below.

Problem 1 (Chawla and Rao [17]). We have

$$
y^{\prime \prime}(t)=-100 y(t), \quad y(0)=1, \quad y^{\prime}(0)=-2 .
$$

Exact solution is $y=-(1 / 5) \sin (10 t)+\cos (10 t)$
Problem 2 (Attili et al. [18]). We have

$$
y^{\prime \prime}(t)=-64 y(t), \quad y(0)=\frac{1}{4}, \quad y^{\prime}(0)=-\frac{1}{2} .
$$

Exact solution is $y=(\sqrt{17} / 16) \sin (8 t+\theta), \theta=\pi-\tan ^{-1}(4)$

Problem 3 (Lambert and Watson [5]). We have

$$
\begin{aligned}
& \frac{d^{2} y_{1}(t)}{d t^{2}}=-v^{2} y_{1}(t)+v^{2} f(t)+f^{\prime \prime}(t), \\
& \frac{d^{2} y_{2}(t)}{d t^{2}}=-v^{2} y_{2}(t)+v^{2} f(t)+f^{\prime \prime}(t), \\
& y_{1}(0)=a+f(0), \quad y_{1}^{\prime}(0)=f^{\prime}(0), \\
& y_{2}(0)=f(0), \quad y_{2}^{\prime}(0)=v a+f^{\prime}(0) .
\end{aligned}
$$

Exact solution is $y_{1}(t)=a \cos (v t)+f(t), y_{2}(t)=a \sin (v t)+$ $f(t)$, and $f(t)$ is chosen to be $e^{-0.05 t}$ and parameters $v$ and $a$ are 20 and 0.1 respectively.

Problem 4 (an almost periodic orbit problem given in Stiefel and Bettis [19]). We have

$$
\begin{gathered}
y_{1}^{\prime \prime}(t)+y_{1}(t)=0.001 \cos (t), \\
y_{1}(0)=1, \quad y_{1}^{\prime}(0)=0, \\
y_{2}^{\prime \prime}(t)+y_{2}(t)=0.001 \sin (t), \\
y_{2}(0)=0, \quad y_{2}^{\prime}(0)=0.9995 .
\end{gathered}
$$

Exact solution is $y_{1}=\cos (t)+0.0005 t \sin (t), y_{2}=\sin (t)-$ $0.0005 t \cos (t)$.

Problem 5 (inhomogeneous system studied by Franco [1]). We have

$$
\begin{gathered}
y^{\prime \prime}(t)+\left(\begin{array}{cc}
13 & -12 \\
-12 & 13
\end{array}\right) y(t)=\left(\begin{array}{c}
g_{1}(t) \\
g_{2}(t)
\end{array}\right), \\
y(0)=\left(\begin{array}{l}
1 \\
0
\end{array}\right), \quad y^{\prime}(0)=\left(\begin{array}{c}
-4 \\
8
\end{array}\right), \\
g_{1}(t)=9 \cos (2 t)-12 \sin (2 t), \\
g_{2}(t)=-12 \cos (2 t)+9 \sin (2 t)
\end{gathered}
$$

Exact solutions are

$$
y(t)=\left(\begin{array}{c}
\sin (t)-\sin (5 t)+\cos (2 t) \\
\sin (t)+\sin (5 t)+\sin (2 t)
\end{array}\right)
$$




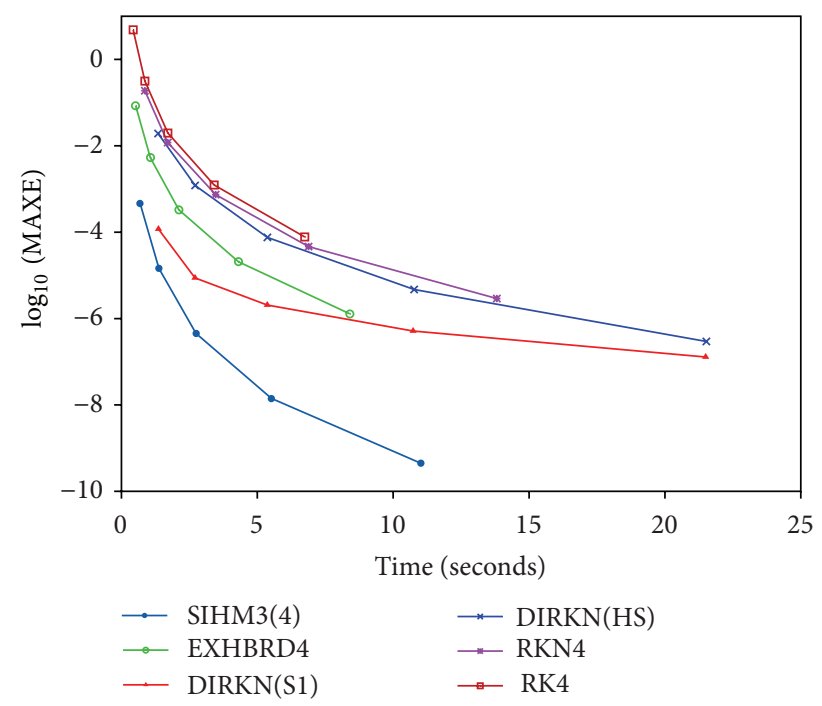

Figure 1: The efficiency curves for SIHM3(4) method for Chawla and Rao problem with $h=0.125 / 2^{i}, i=3, \ldots, 7$.

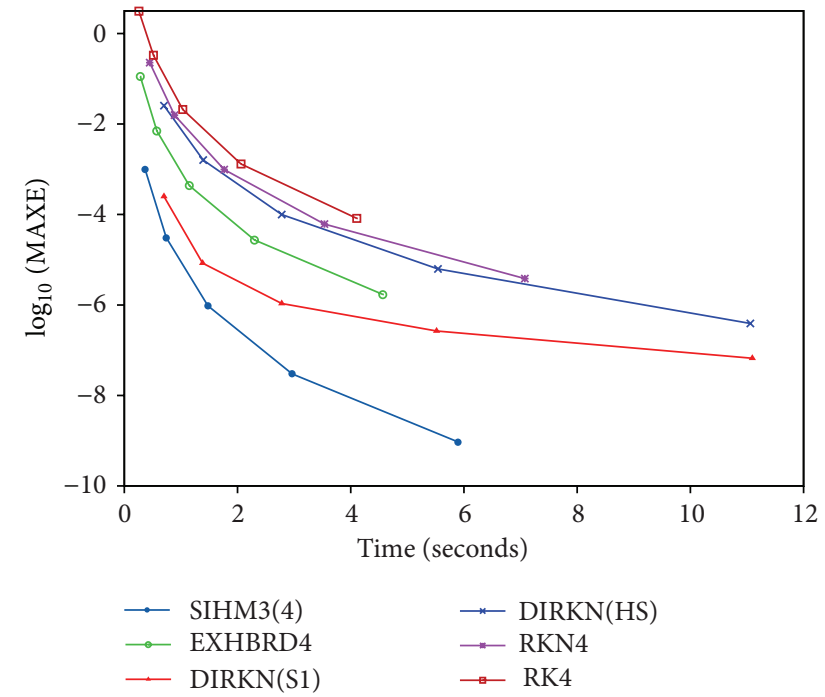

Figure 2: The efficiency curves for SIHM3(4) method for Attili problem with $h=0.5 / 2^{i}, i=1, \ldots, 5$.

Problem 6 (Allen and Wing [20]). We have

$$
y^{\prime \prime}(t)=-y(t)+t, \quad y(0)=1, \quad y^{\prime}(0)=2 .
$$

Exact solution is $y=\sin (t)+\cos (t)+t$.

Problem 7 (inhomogeneous problem studied by Papadopoulos et al. [21]). We have

$$
\begin{gathered}
y^{\prime \prime}(t)=-v^{2} y(t)+\left(v^{2}-1\right) \sin (t), \\
y(0)=1, \quad y^{\prime}(0)=v+1,
\end{gathered}
$$

where $v \gg 1$.

Exact solution is $y(t)=\cos (v t)+\sin (v t)+\sin (t)$. Numerical result is for the case $v=10$.

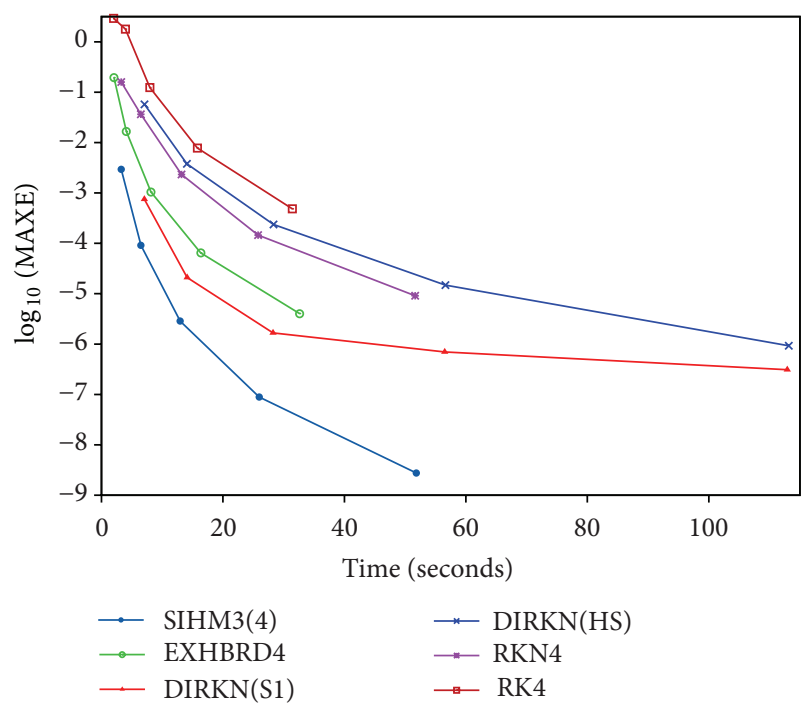

Figure 3: The efficiency curves for SIHM3(4) method for Lambert and Watson problem with $h=0.5 / 2^{i}, i=1, \ldots, 5$.

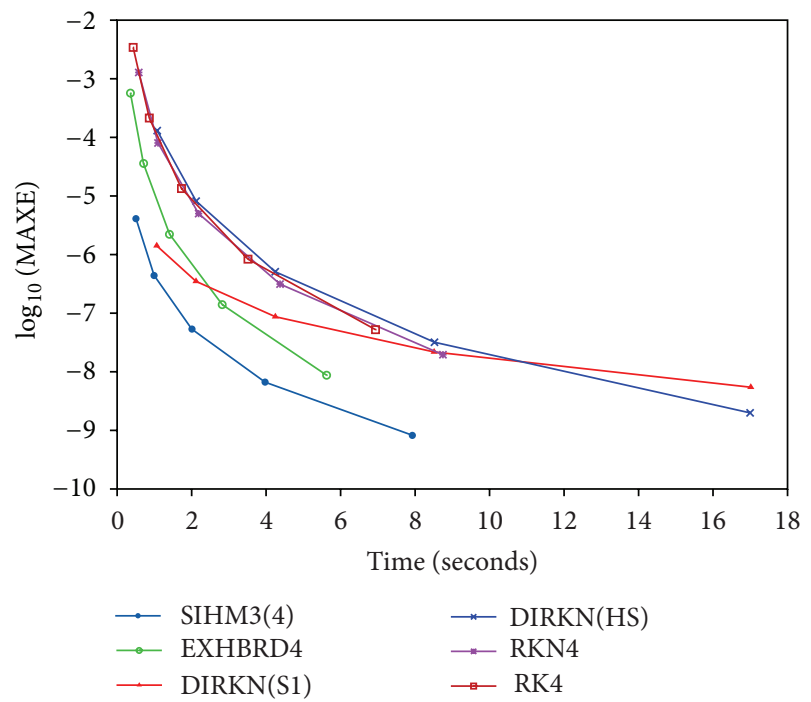

FIgURE 4: The efficiency curves for SIHM3(4) method for An almost Periodic Orbit problem with $h=0.125 / 2^{i}, i=1, \ldots, 5$.

Problem 8 (orbital problem studied by van der Houwen and Sommeijer [22]). We have

$$
\begin{aligned}
& y_{1}^{\prime \prime}(t)=-4 t^{2} y_{1}-\frac{2 y_{2}}{\sqrt{y_{1}^{2}+y_{2}^{2}}}, \\
& y_{1}\left(t_{0}\right)=0, \quad y^{\prime}\left(t_{0}\right)=-\sqrt{2 \pi}, \\
& y_{2}^{\prime \prime}(t)=-4 t^{2} y_{2}+\frac{2 y_{1}}{\sqrt{y_{1}^{2}+y_{2}^{2}}}, \\
& y_{2}\left(t_{0}\right)=1, \quad y^{\prime}\left(t_{0}\right)=0, \quad \sqrt{\frac{\pi}{2}} \leq t \leq t_{\text {end }} .
\end{aligned}
$$

Exact solution is $y_{1}(t)=\cos \left(t^{2}\right), y_{2}(t)=\sin \left(t^{2}\right)$ 


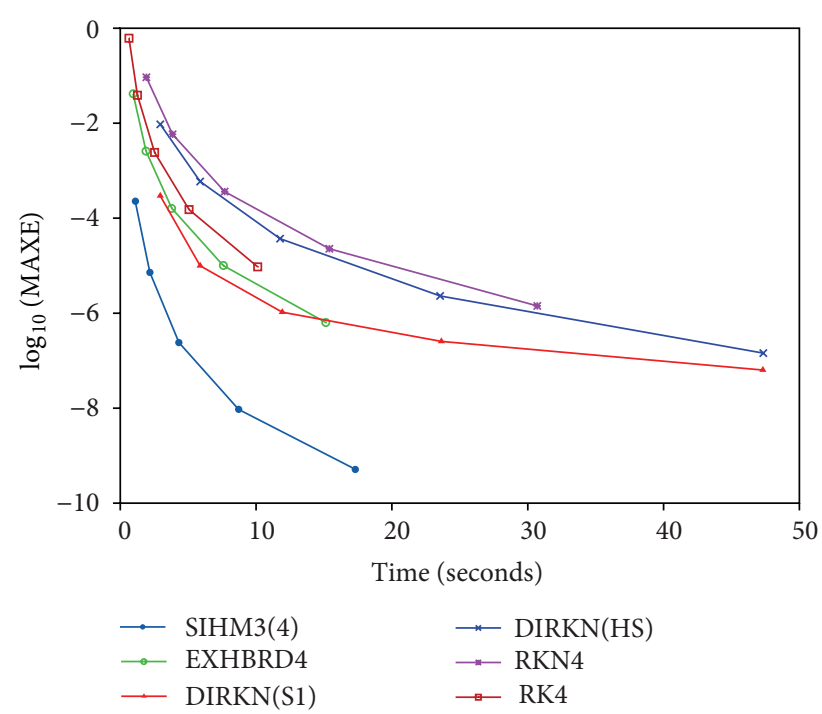

FIgURE 5: The efficiency curves for SIHM3(4) method for Inhomogeneous system with $h=0.125 / 2^{i}, i=1, \ldots, 5$.

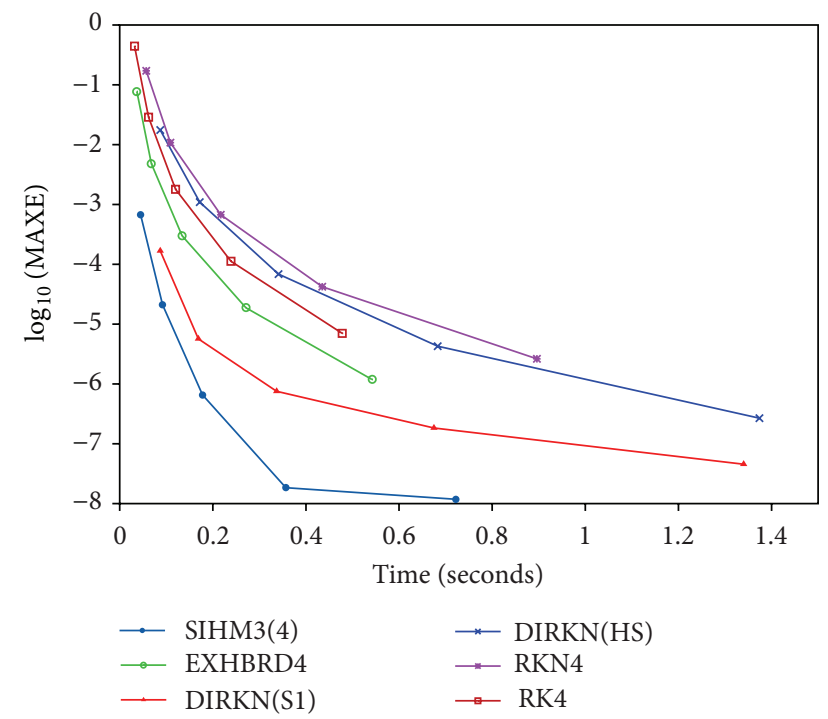

FIgURE 6: The efficiency curves for SIHM3(4) method for Allen and Wing problem with $h=0.125 / 2^{i}, i=2, \ldots, 6$.

The following notations are used in Figures 1-16.

(i) SIHM3(4): a semi-implicit hybrid method of order four with dispersive order six and zero dissipation.

(ii) SIHM3(5): a semi-implicit hybrid method of order five with dispersive order six and zero dissipation.

(iii) SIHM4(5): a semi-implicit hybrid method of order five with dispersive order eight and dissipative order five.

(iv) $\operatorname{DIRKN}(\mathrm{S} 1)$ : a three-stage fourth-order dispersive order six method with "small" dissipation constant

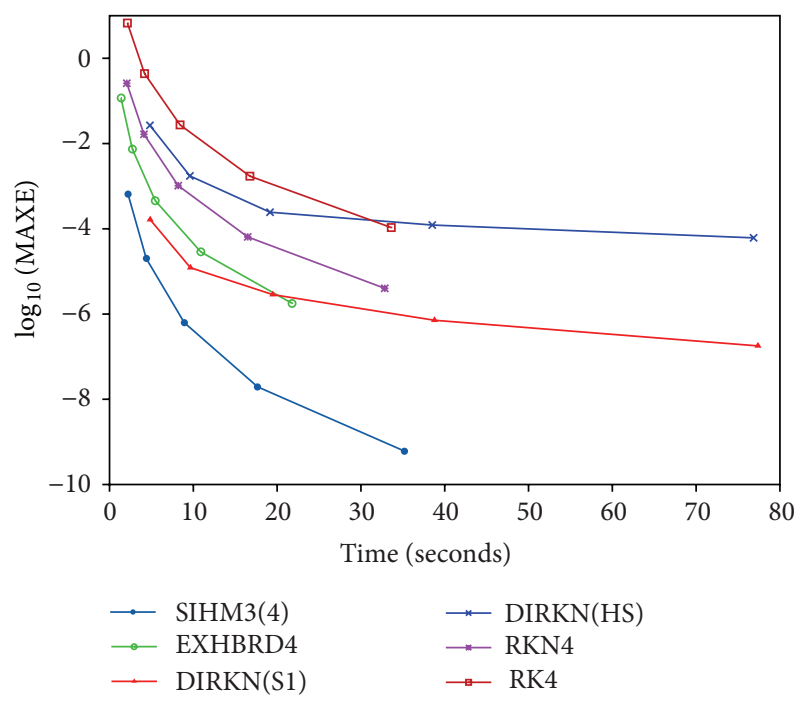

FIgURE 7: The efficiency curves for SIHM3(4) method for Inhomogeneous problem with $h=0.125 / 2^{i}, i=2, \ldots, 6$.

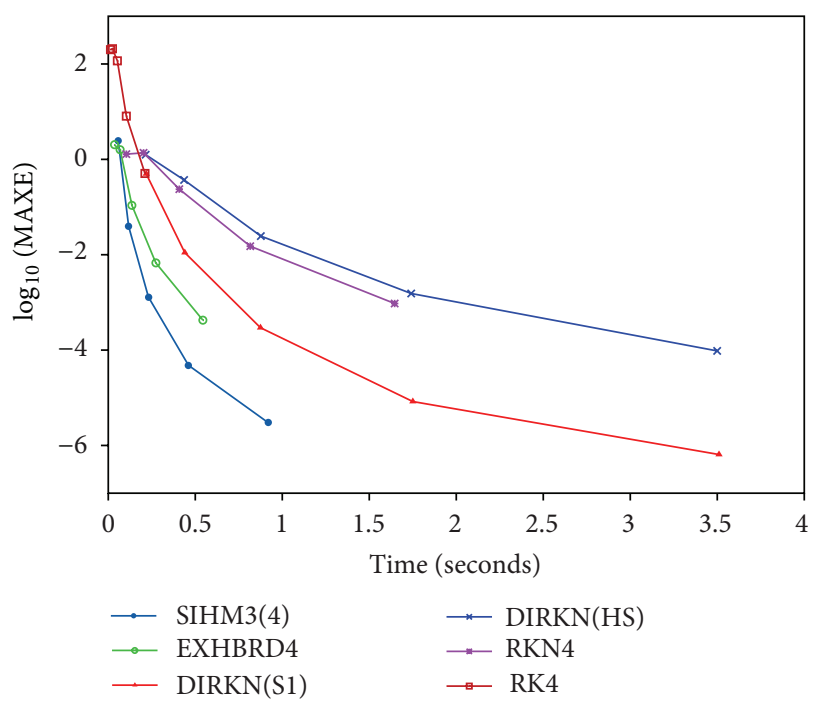

FIgURE 8: The efficiency curves for SIHM3(5) and SIHM4(5) methods for Orbital problem with $h=0.125 / 2^{i}, i=4, \ldots, 8$.

and principal local truncation errors derived by Senu et al. [7].

(v) DIRKN(HS): a three-stage fourth-order derived by Sommeijer [13].

(vi) $\operatorname{DIRKN}(\mathrm{S} 2)$ : a four-stage fourth-order dispersive order eight method with "small" dissipation constant derived by Senu et al. [10].

(vii) RKN4: a classical RKN method order four in [14].

(viii) RK4: a classical RK method order four in [14].

(ix) RKN5: a five-stage fifth-order RKN method derived by Butcher [16].

(x) RK6: a seven-stage sixth-order RK method derived by Hairer et al. [15]. 


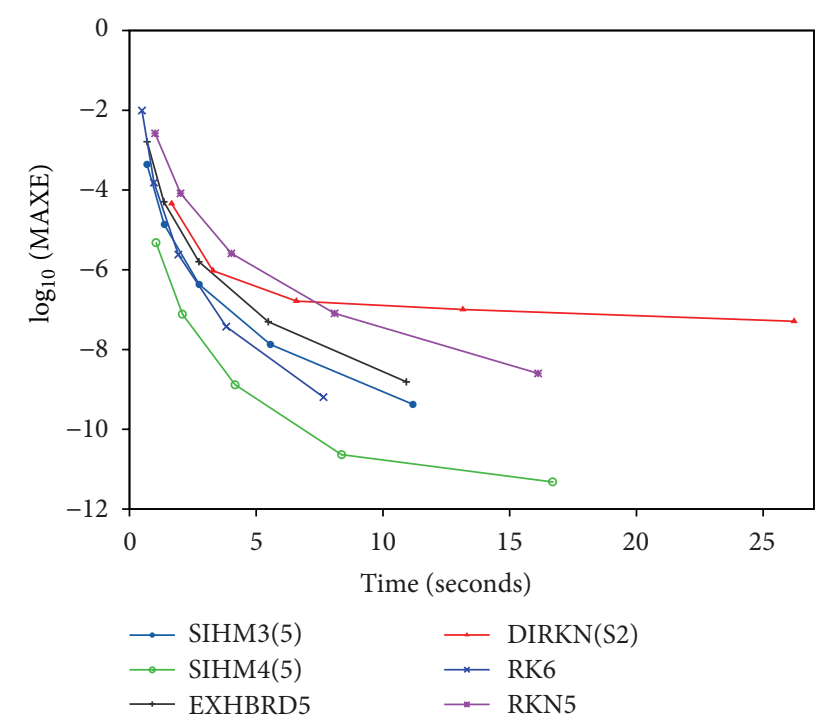

FIGURE 9: The efficiency curves for SIHM3(5) and SIHM4(5) methods for Chawla and Rao problem with $h=0.125 / 2^{i}, i=$ $3, \ldots, 7$.

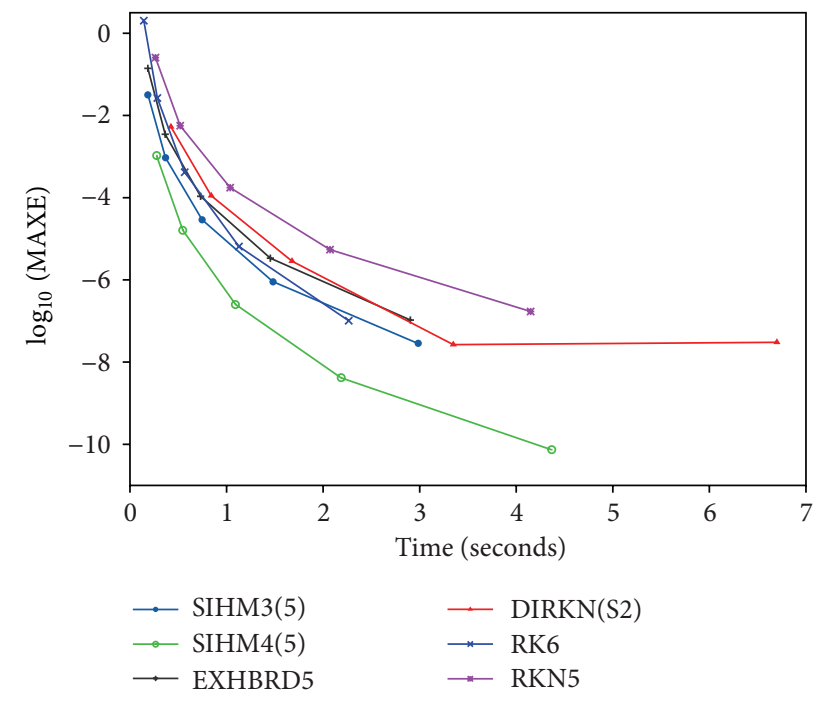

FIgURE 10: The efficiency curves for SIHM3(5) and SIHM4(5) methods for Attili problem with $h=0.125 / 2^{i}, i=1, \ldots, 5$.

(xi) EXHBRD4: a explicit three-stage fourth-order hybrid method derived by Franco [3].

(xii) EXHBRD5: a explicit four-stage fifth-order hybrid method derived by Franco [3].

In order to evaluate the effectiveness of the semi-implicit hybrid methods, we solved several problems which have oscillatory solutions. To make a comparison of SIHM and other existing methods, one measure of the accuracy is examined using the absolute error which is defined by

$$
\text { Absolute error }=\max \left\{\left|y\left(t_{n}\right)-y_{n}\right|\right\},
$$

where $y\left(t_{n}\right)$ is the exact solution and $y_{n}$ is the computed solution.

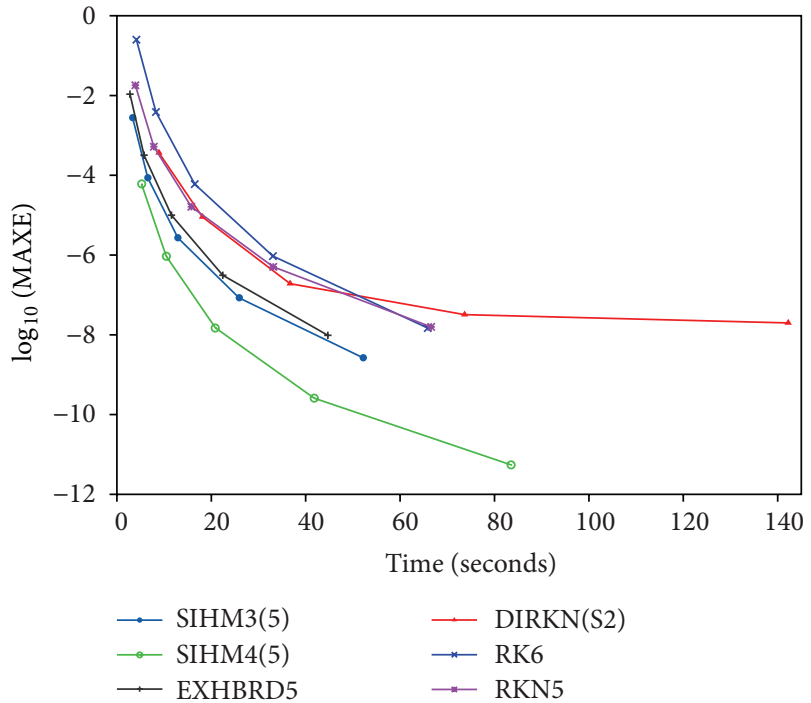

Figure 11: The efficiency curves for SIHM3(5) and SIHM4(5) methods for Lambert and Watson problem with $h=0.125 / 2^{i}, i=$ $1, \ldots, 5$.

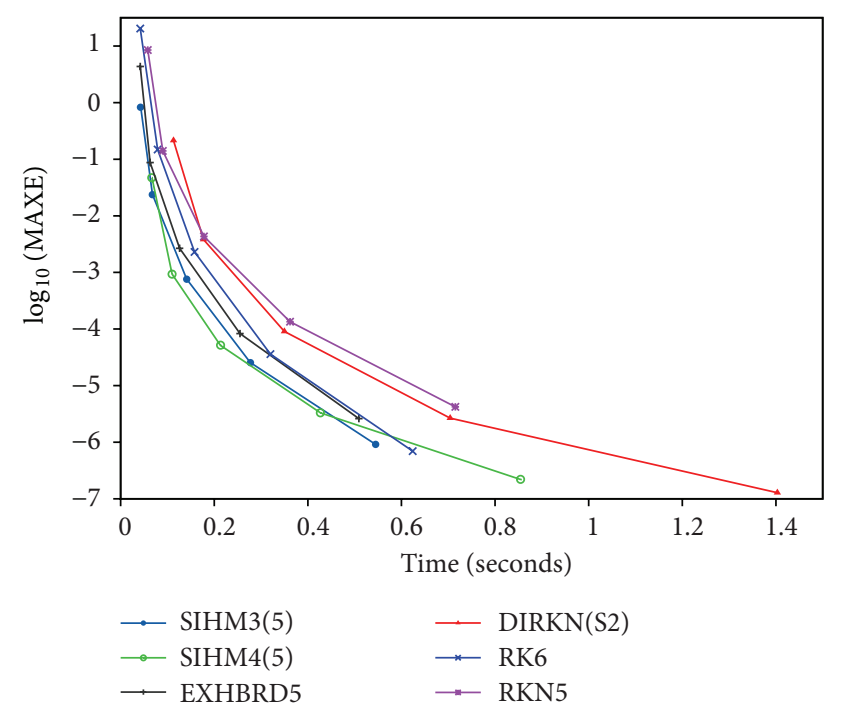

FIGURE 12: The efficiency curves for SIHM3(5) and SIHM4(5) methods for An almost Periodic Orbit problem with $h=0.125 / 2^{i}, i=$ $1, \ldots, 5$.

For comparison purposes, in analyzing the numerical results, methods of the same order will be compared. The results are given in Figures 1-16. We present the efficiency curves where the common logarithm of the maximum global error along the integration versus the CPU time is taken. From Figures 1, 2, 3, 4, 5, 6, 7, and 8, we observed that the new SIHM3(4) is the most efficient for integrating second-order differential equations possessing oscillatory solutions, followed by diagonally implicit DIRKN(S1), original explicit hybrid method EXHBRD4, and other methods like DIRKN(HS), RKN4, and RK4. 


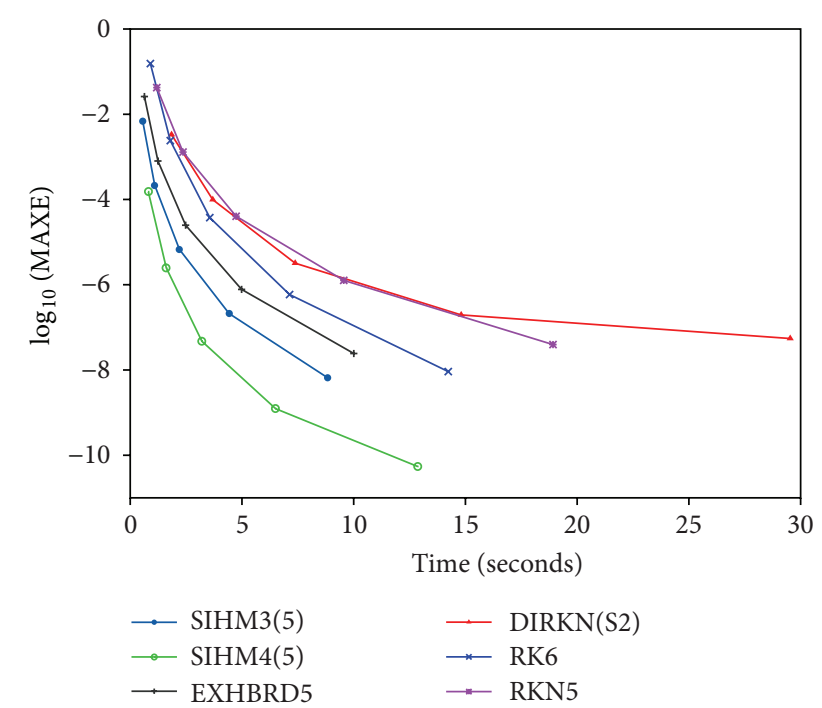

FIgURE 13: The efficiency curves for SIHM3(5) and SIHM4(5) methods for Inhomogeneous system with $h=0.9 / 2^{i}, i=1, \ldots, 5$.

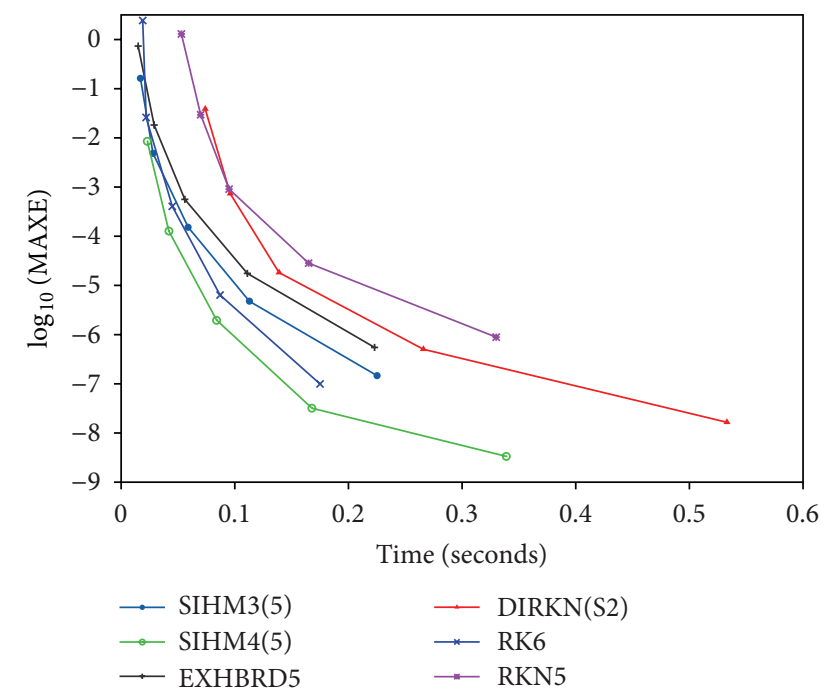

FIGURE 14: The efficiency curves for SIHM3(5) and SIHM4(5) methods for Allen and Wing problem with $h=0.125 / 2^{i}, i=$ $1, \ldots, 5$.

From Figures 9, 10, 11, 12, 13, 14, 15, and 16, for the fifth-order methods we observed that SIHM4(5) is the most efficient, followed by SIHM3(5) and EXHBRD5 and the rest of the methods. Even though the new methods are semiimplicit and fairly expensive in terms of time consumed, they are still more efficient compared to the explicit counterpart.

\section{Conclusion}

In this paper three-stage semi-implicit hybrid methods of order four and five are developed and denoted by SIHM3(4) and SIHM3(5), respectively, they have dispersion order six and zero dissipation. We also developed method of four-stage and fifth order denoted by SIHM4(5) which has dispersion

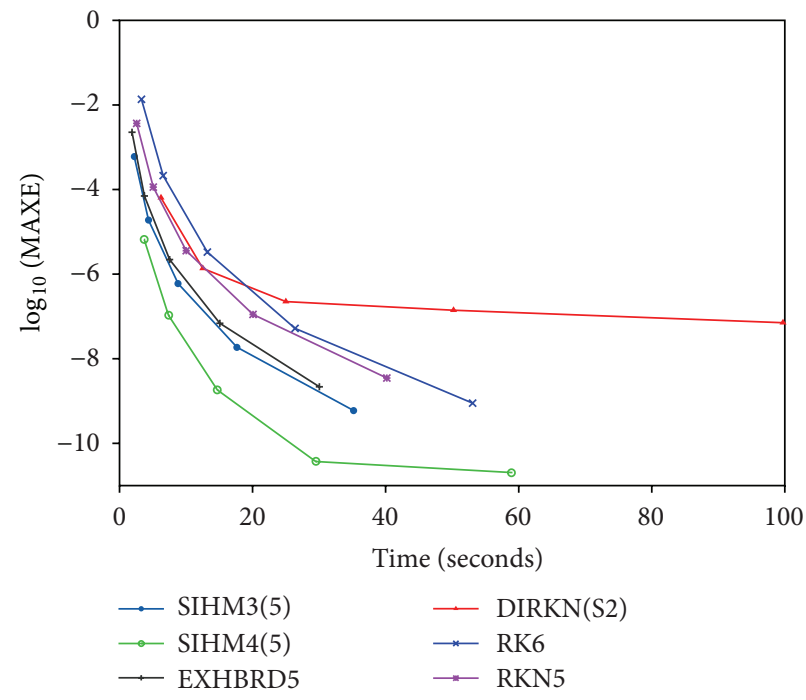

FIgURE 15: The efficiency curves for SIHM3(5) and SIHM4(5) methods for Inhomogeneous problem with $h=0.125 / 2^{i}, i=$ $2, \ldots, 6$.

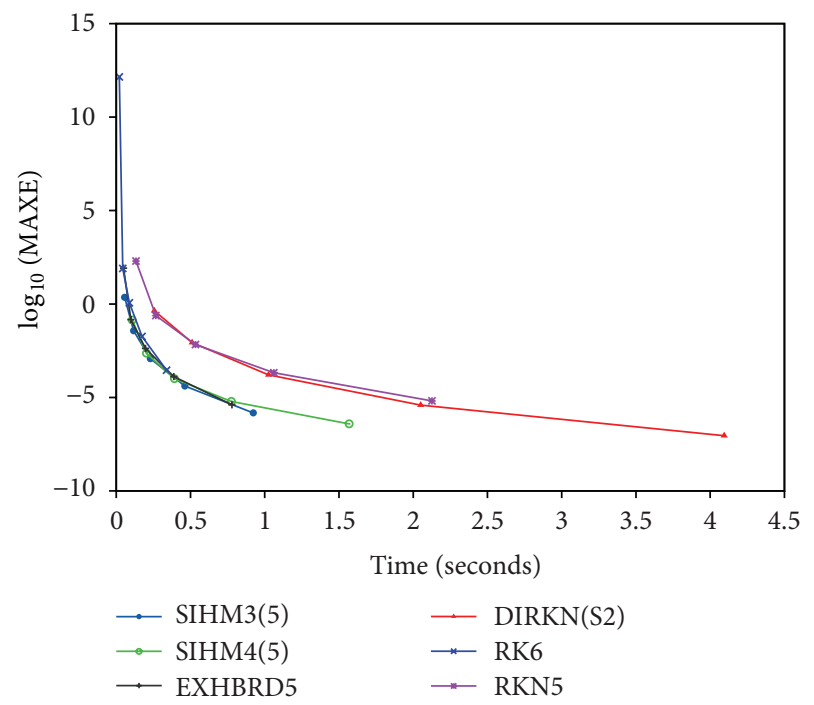

FIGURE 16: The efficiency curves for SIHM3(5) and SIHM4(5) methods for Orbital problem with $h=0.125 / 2^{i}, i=4, \ldots, 8$.

order eight and dissipation order five. All the three methods developed are suitable for solving second-order IVPs which are oscillatory in nature. From the efficiency curves shown in Figures 1-16, we can conclude that all the methods are very efficient compared to the well-known existing methods of the same order in the scientific literature.

\section{References}

[1] J. M. Franco, "An explicit hybrid method of Numerov type for second-order periodic initial-value problems," Journal of Computational and Applied Mathematics, vol. 59, no. 1, pp. 7990, 1995. 
[2] L. K. Yap, F. Ismail, M. Suleiman, and S. Md. Amin, "Block methods based on Newton interpolations for solving special second order ordinary differential equations directly," Journal of Mathematics and Statistics, vol. 4, no. 3, pp. 174-180, 2008.

[3] J. M. Franco, "A class of explicit two-step hybrid methods for second-order IVPs," Journal of Computational and Applied Mathematics, vol. 187, no. 1, pp. 41-57, 2006.

[4] J. P. Coleman, "Order conditions for a class of two-step methods for $y^{\prime \prime}=f(x, y)$," IMA Journal of Numerical Analysis, vol. 23, no. 2, pp. 197-220, 2003.

[5] J. D. Lambert and I. A. Watson, "Symmetric multistep methods for periodic initial value problems," Journal of the Institute of Mathematics and Its Applications, vol. 18, no. 2, pp. 189-202, 1976.

[6] H. Van de Vyver, "A symplectic Runge-Kutta-Nyström method with minimal phase-lag," Physics Letters A, vol. 367, no. 1-2, pp. 16-24, 2007.

[7] N. Senu, M. Suleiman, F. Ismail, and M. Othman, "A singly diagonally implicit Runge-Kutta-Nyström method for solving oscillatory problems," IAENG International Journal of Applied Mathematics, vol. 41, no. 2, pp. 155-161, 2011.

[8] L. Brusa and L. Nigro, "A one-step method for direct integration of structural dynamic equations," International Journal for Numerical Methods in Engineering, vol. 15, no. 5, pp. 685-699, 1980.

[9] P. J. van der Houwen and B. P. Sommeijer, "Explicit Runge-Kutta (-Nyström) methods with reduced phase errors for computing oscillating solutions," SIAM Journal on Numerical Analysis, vol. 24, no. 3, pp. 595-617, 1987.

[10] N. Senu, M. Suleiman, F. Ismail, and M. Othman, "A fourthorder diagonally implicit Runge-Kutta- Nyström method with dispersion of high order," in Proceedings of the 4th International Conference on Applied Mathematics, Simulation, Modelling (ASM '10), pp. 78-82, July 2010.

[11] A. A. Kosti, Z. A. Anastassi, and T. E. Simos, "Construction of an optimized explicit Runge-Kutta-Nyström method for the numerical solution of oscillatory initial value problems," Computers \& Mathematics with Applications, vol. 61, no. 11, pp. 3381-3390, 2011.

[12] A. A. Kosti, Z. A. Anastassi, and T. E. Simos, "An optimized explicit Runge-Kutta-Nyström method for the numerical solution of orbital and related periodical initial value problems," Computer Physics Communications, vol. 183, no. 3, pp. 470-479, 2012.

[13] B. P. Sommeijer, "A note on a diagonally implicit RungeKutta-Nyström method," Journal of Computational and Applied Mathematics, vol. 19, no. 3, pp. 395-399, 1987.

[14] J. R. Dormand, Numerical Methods for Differential Equations, Library of Engineering Mathematics, CRC Press, Boca Raton, Fla, USA, 1996.

[15] E. Hairer, S. P. Nørsett, and G. Wanner, Solving Ordinary Differential Equations 1, Springer, Berlin, Germany, 2010.

[16] J. C. Butcher, Numerical Methods for Ordinary Differential Equations, John Wiley \& Sons, Chichester, UK, 2nd edition, 2008.

[17] M. M. Chawla and P. S. Rao, "High-accuracy P-stable methods for $y^{\prime \prime}=f(x, y)$," IMA Journal of Numerical Analysis, vol. 5, no. 2, pp. 215-220, 1985.

[18] B. S. Attili, K. Furati, and M. I. Syam, "An efficient implicit Runge-Kutta method for second order systems," Applied Mathematics and Computation, vol. 178, no. 2, pp. 229-238, 2006.
[19] E. Stiefel and D. G. Bettis, "Stabilization of Cowell's method," Numerische Mathematik, vol. 13, pp. 154-175, 1969.

[20] R. C. Allen, Jr. and G. M. Wing, "An invariant imbedding algorithm for the solution of inhomogeneous linear two-point boundary value problems," Journal of Computational Physics, vol. 14, pp. 40-58, 1974.

[21] D. F. Papadopoulos, Z. A. Anastassi, and T. E. Simos, "A phasefitted Runge-Kutta-Nyström method for the numerical solution of initial value problems with oscillating solutions," Computer Physics Communications, vol. 180, no. 10, pp. 1839-1846, 2009.

[22] P. J. van der Houwen and B. P. Sommeijer, "Diagonally implicit Runge-Kutta-Nyström methods for oscillatory problems," SIAM Journal on Numerical Analysis, vol. 26, no. 2, pp. 414-429, 1989. 


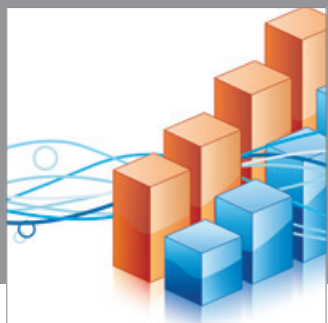

Advances in

Operations Research

mansans

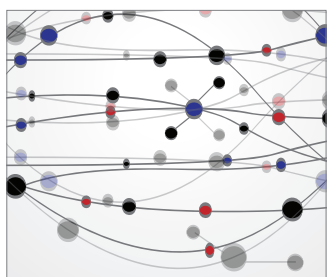

The Scientific World Journal
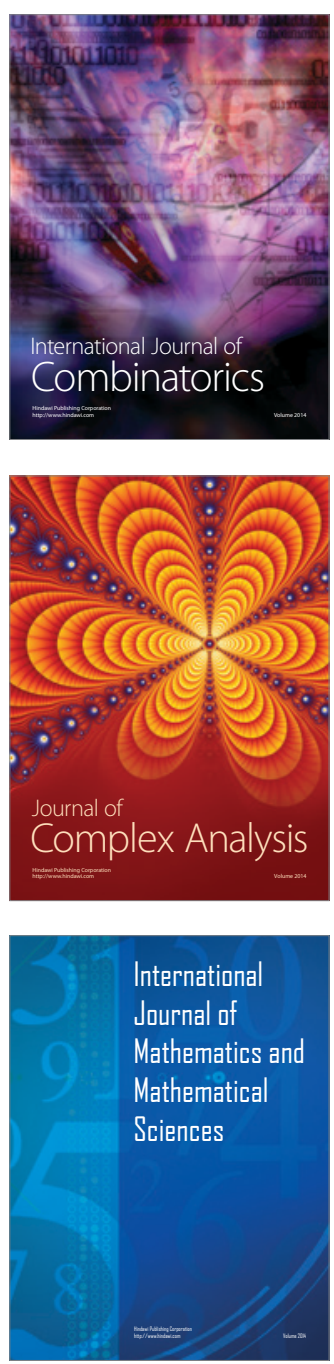
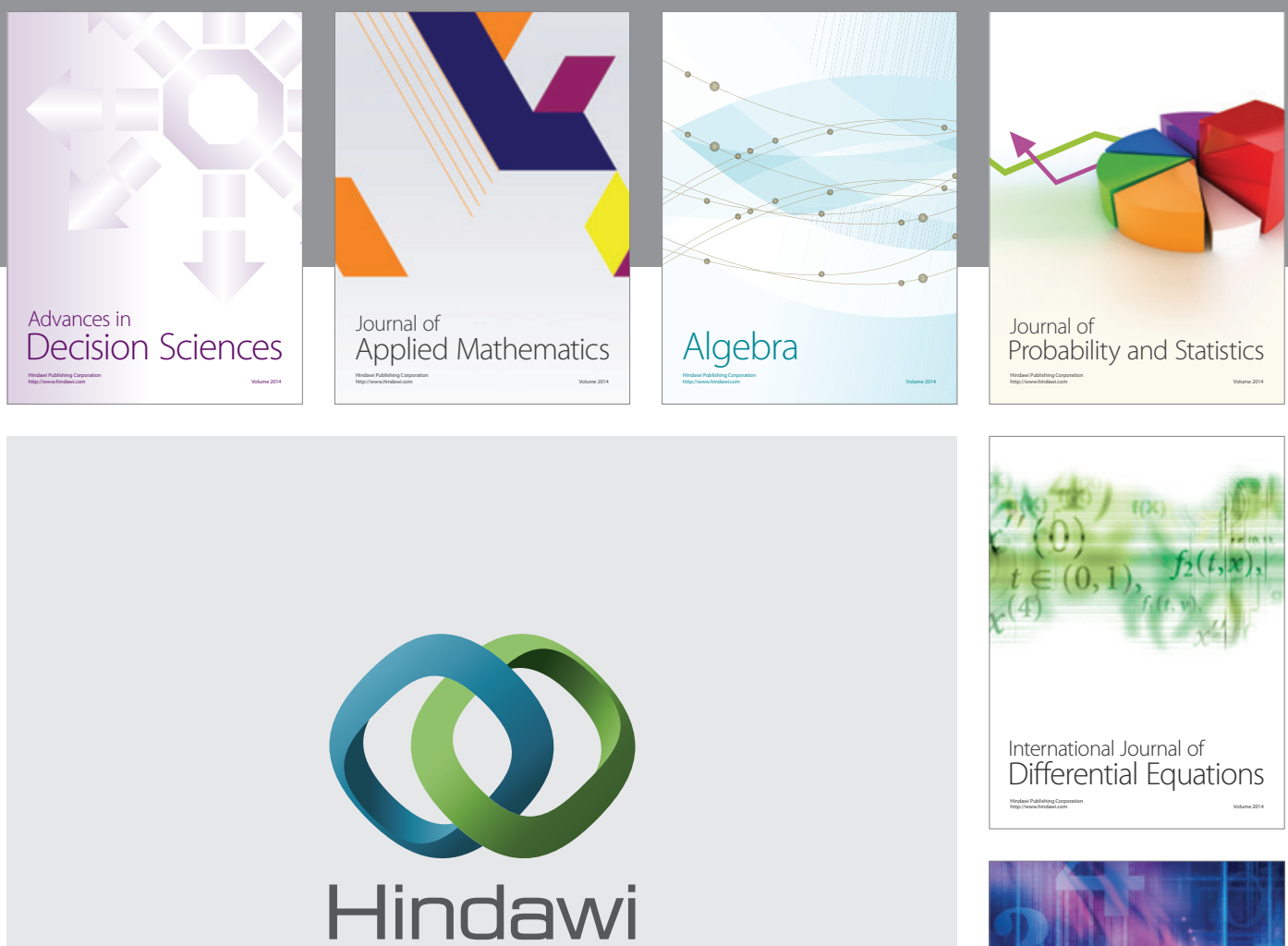

Submit your manuscripts at http://www.hindawi.com
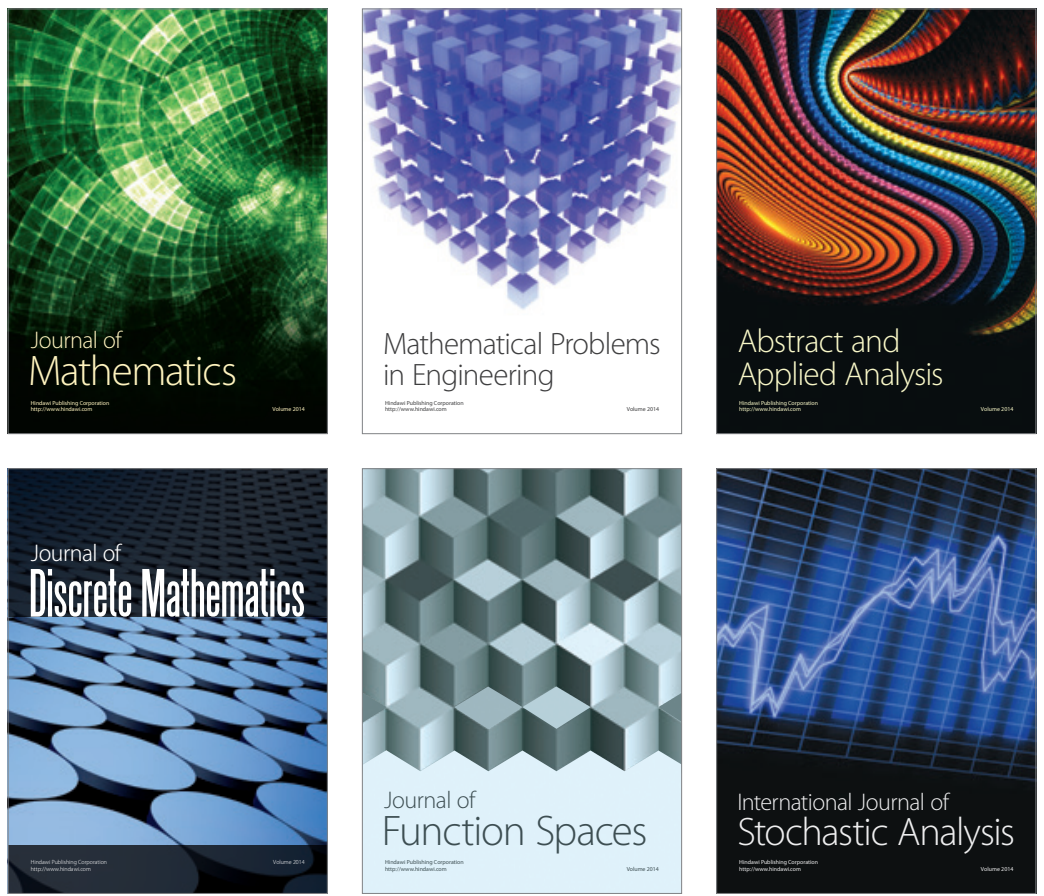

Journal of

Function Spaces

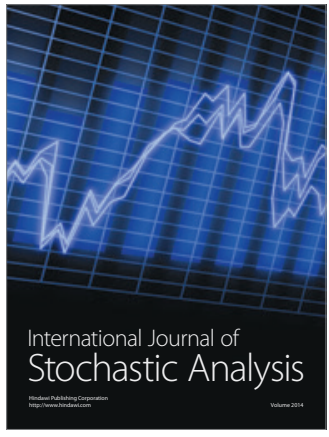

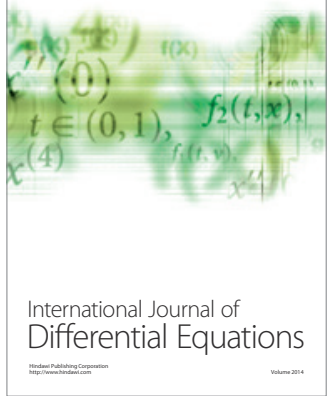
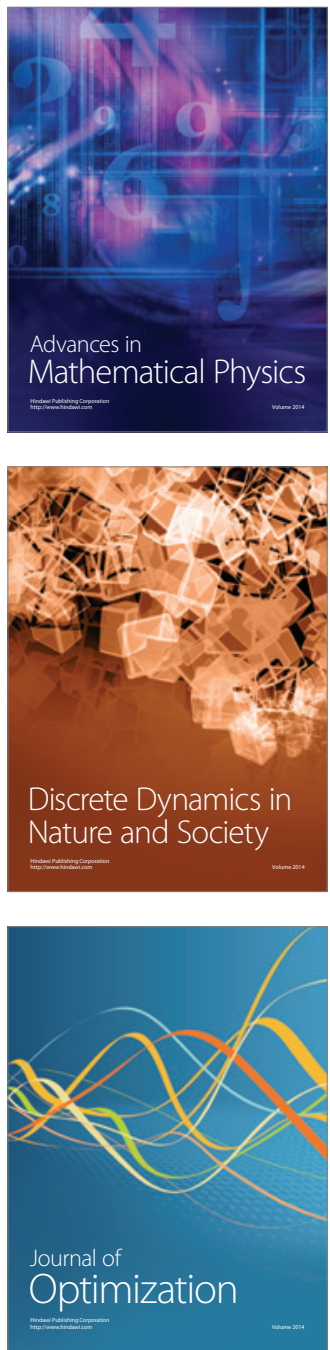